\title{
Cuisine chypriote et cuisine grecque
}

Yiannis E. IOANNOU

\section{(2) OpenEdition \\ Journals}

Édition électronique

URL : http://journals.openedition.org/transtexts/595

DOI : 10.4000/transtexts.595

ISSN : 2105-2549

Éditeur

Gregory B. Lee

Référence électronique

Yiannis E. IOANNOU, « Cuisine chypriote et cuisine grecque », Transtext(e)s Transcultures 跨文本跨文 化 [En ligne], 10 | 2015, mis en ligne le 21 juin 2016, consulté le 03 mai 2019. URL : http://

journals.openedition.org/transtexts/595; DOI : 10.4000/transtexts.595

Ce document a été généré automatiquement le 3 mai 2019.

(c) Tous droits réservés 


\title{
Cuisine chypriote et cuisine grecque
}

\author{
Yiannis E. IOANNOU
}

1 Le présent article tente une première approche du phénomène de la présence à Chypre d'un nombre croissant de restaurants et de bistrots grecs, notamment durant la dernière quinzaine d'années. Il semblerait que ce phénomène, à part l'enrichissement de la cuisine chypriote souvent différente de celle du continent grec, permettrait aussi la concrétisation de la part de la clientèle chypriote d'un sentiment refoulé depuis des décennies, et notamment depuis l'invasion de la Turquie en 1974, qui est celui de l'union avec la Grèce.

2 A Chypre, ces dernières années et notamment depuis 2009, on remarque que les restaurants à menu grec se multiplient et que les Chypriotes s'intéressent de plus en plus à la cuisine grecque. Paradoxalement, bien que cette période coïncide avec le début de la crise financière, d'abord en Grèce, puis à partir de 2012 à Chypre, le goût pour la cuisine grecque et le succès des restaurants proposant exclusivement une cuisine grecque sur l'île ne semblent pas correspondre à des critères financiers.

3 La majorité de ces restaurants sont tenus par des propriétaires chypriotes qui emploient souvent des employés chypriotes et grecs et la décision d'ouvrir de tels restaurants semble reposer sur une volonté de proposer quelque chose de différent, une cuisine se différenciant de leurs habitudes culinaires. C'est ce qui explique, sans doute, que la plupart des menus proposés ne présentent pas de croisements entre la cuisine chypriote et la cuisine grecque. Au contraire, ces restaurants présentent des repas gastronomiques préparés en utilisant exclusivement des produits grecs. En réalité, il ne s'agit pas (uniquement) de plats traditionnels grecs, mais de plats assez inventifs et originaux, souvent créés par des chefs grecs célèbres. Dès lors, les Chypriotes prennent contact avec une cuisine grecque à la fois familière et tout à fait nouvelle, une cuisine grecque en quelque sorte « réinventée ».

4 Les propriétaires des restaurants questionnés affirment que leurs menus s'adressent à un public large et qu'ils ont pris soin de proposer un choix varié au niveau des prix. En effet, tandis que les propriétaires déclarent que la crise financière n'a pas affecté leur chiffre d'affaires, ils remarquent, pourtant, que les Chypriotes ne passent pas de commandes 
comme autrefois, mais qu'ils prennent de plus en plus en considération l'addition finale : ils commandent moins de plats et préfèrent prendre un verre de vin au lieu de commander une bouteille.

5 Notre enquête, fondée notamment sur un questionnaire puisqu'il n'existe pas d'enquêtes officielles de la part du Ministère du Commerce ou d'autres organismes qui permettraient d'analyser des éléments statistiques, a révélé que le succès de ces restaurants dépend largement de leur localisation. La plupart des restaurants grecs se trouvent en effet à Nicosie, la capitale, et ce sont précisément les propriétaires des restaurants à Nicosie qui déclarent que la crise financière n'a influencé ni le succès de leurs restaurants, ni leur chiffre d'affaires. De plus, la clientèle à Nicosie repose sur un public en majorité chypriote, contrairement aux villes côtières qui s'adressent surtout aux touristes. Ces restaurants ne sont pas affectés par la baisse ou par la hausse du tourisme. De plus, ces dernières années, Nicosie connaît un développement considérable : les quartiers de la vieille ville sont rénovés et attirent un public assez varié (étudiants, artistes et intellectuels etc.). L'architecture et le décor de ces restaurants témoignent du fait que les propriétaires cherchent à attirer un public « sélectif » mais non pas forcément un public d'« élite ».

6 Les mêmes données semblent être valables pour Limassol, ville qui combine une clientèle chypriote et une clientèle touristique, mais aussi une communauté russe assez aisée. Limassol est réputée être une ville de fête où les habitants ont l'habitude de sortir plus souvent que ceux des autres villes. On peut y trouver plusieurs restaurants à menus exclusivement grecs, mais aussi des restaurants à menus qui combinent des spécialités chypriotes et des spécialités grecques. Ce dernier point s'explique précisément par le fait que ces restaurants s'adressent aussi à des touristes qui souhaitent connaitre la cuisine chypriote, mais s'explique aussi par le fait qu'il existe une clientèle chypriote plus conservatrice qui reste « fidèle » à ses habitudes culinaires.

7 C'est ce que l'on observe également dans les lieux touristiques par excellence que sont Ayia Napa et Protaras au sud-est de l'île. Dans cette région, les restaurants proposent des menus qui marient la cuisine chypriote avec la cuisine grecque, puisqu'ils s'adressent à la fois à une clientèle chypriote minoritaire et à une clientèle massivement touristique qui souhaite connaitre la gastronomie de deux pays ou qui inconsciemment confond la cuisine chypriote avec la cuisine grecque. Pourtant, on y trouve des restaurants offrant des mets gastronomiques où la cuisine chypriote est elle aussi "revisitée " et " réinventée ». La cuisine chypriote, assez riche mais parfois lourde, devient en effet raffinée une fois revisitée et recréée à partir d'un croisement entre les deux traditions.

La situation change dans des régions plus touchées par la crise financière comme dans le cas de Larnaca. Dans cette ville, la crise financière a provoqué une baisse du nombre des touristes, mais aussi une baisse de la clientèle chypriote. Cependant, en interrogeant les propriétaires des restaurants grecs, on réalise vite que ce n'est pas uniquement la crise financière qui a provoqué une chute dans leur chiffre d'affaires, mais aussi le fait que la clientèle chypriote de Larnaca est plus conservatrice et affiche une préférence pour des restaurants chypriotes traditionnels de masse, tout en étant aussi attirée par les restaurants grecs de la capitale, les distances n'étant pas dissuasives.

9 Les propriétaires de ces restaurants s'accordent pour dire que ce sont les voyages effectués par les Chypriotes durant les quinze dernières années (notamment à partir de 1998- 1999), période durant laquelle l'économie de l'île avait connu un essor considérable, qui ont permis aux Chypriotes d'entrer en contact avec la cuisine grecque. Ce qui signifie 
que l'ouverture de ces restaurants à Chypre n'est pas uniquement le résultat de la crise financière, mais aussi le fruit d'une expérience culturelle récente. Ces hommes d'affaires ont ensuite investi dans un effet de mode résultant de la redécouverte de la Grèce par le voyage. Ce n'est pas un hasard si de nombreux restaurants proposent également des soirées, notamment durant le weekend, agrémentées de musique grecque. Le contact des Chypriotes avec la cuisine et la musique grecques dans une ambiance et dans un répertoire assez recherchés, et non pas massivement populaires, enrichit les possibilités de choix et ajoute une dimension culturelle bien ancrée dans la tradition de l'hellénisme.

10 À partir des années 90, des émissions grecques matinales ont fleuri à la télévision chypriote, une partie du programme étant consacrée à l'exécution d'une recette par un chef souvent célèbre. Ces dernières années ont vu le développement des émissions télévisées exclusivement culinaires, engouement qui change considérablement les habitudes culinaires.

11 Ces émissions ont eu pour conséquence l'importation croissante de produits grecs dans les supermarchés et les épiceries chypriotes, ce qui n'était pas le cas il y a une vingtaine d'années. On ne doit pas négliger non plus l'essor des livres de recettes grecques dans le marché chypriote, surtout lorsque ceux-ci sont signés par un chef rendu célèbre grâce aux émissions de télévision.

12 Ces différents éléments déterminent non seulement le contact des Chypriotes avec la cuisine grecque, mais aussi leur contact avec la cuisine chypriote. Les propriétaires des restaurants grecs, surtout à Nicosie, affirment que ce contact ne change pas, au fond, leurs habitudes culinaires, surtout dans leur quotidien. Les plats proposés représentent une cuisine raffinée qui a comme conséquence un raffinement dans les habitudes alimentaires chez une minorité de Chypriotes qui prennent conscience que le plus important n'est pas la quantité, caractéristique des restaurants typiquement chypriotes, mais la qualité. Ce nouveau comportement se situe à l'opposé des restaurants chypriotes traditionnels, où les fameux 'mézédès' se rapprochent plutôt des descriptions rabelaisiennes.

13 La crise a toutefois favorisé l'arrivée assez massive des Grecs spécialisés, entre autres, dans la restauration et l'hôtellerie. En général, on peut dire que le domaine de la gastronomie présente un paradoxe. En pleine période de crise, les émissions de télévision culinaires connaissent un grand succès, le marché du livre de recettes est assez prometteur tandis que la gamme des produits alimentaires s'élargit. De plus, les restaurants grecs à Chypre marchent, selon les propriétaires, plutôt bien. Comment peuton, alors, expliquer ce phénomène? Il semblerait que le domaine de la gastronomie évoque le souvenir d'une période de prospérité auquel on tient et que l'on souhaite préserver l'une des dernières traces d'une société de consommation devenue de plus en plus sélective.

14 Cet article ne peut s'achever sans l'évocation d'une certaine tendance visible dans la clientèle chypriote de ces restaurants. En réalité, au fond de chaque Chypriote, malgré les développements politiques, il existe encore des traces d'un rêve politique manqué, celui de l'union de l'île avec la Grèce, comme la Crète au début du $20^{\mathrm{e}}$ siècle, le Dodécanèse après la deuxième guerre etc. La réapparition, depuis la décennie 1990, d'un discours national et même nationaliste, disparu provisoirement à cause des évènements de 1974, a ravivé le sentiment de l'appartenance nationale chez une partie de la classe moyenne et de la bourgeoisie nationaliste de l'île. Pour cette couche de la population, le fait de fréquenter des restaurants de cuisine grecque et si possible, avec des employés venant du 
continent, acquiert une dimension symbolique importante puisqu'il suggère une identification, ne serait-ce qu'au niveau culinaire avec 'la mère patrie'. Cette pratique soulagerait d'une part le sentiment d'échec par rapport à la réalisation des aspirations nationales de l'île; elle apaiserait d'autre part un fort sentiment d'injustice, sentiment éprouvé par les Chypriotes durant les quarante dernières années à l'égard d'une communauté internationale ne s'étant, selon eux, jamais mobilisée suffisamment pour imposer des sanctions à la Turquie qui continue, aujourd'hui encore, d'occuper la partie nord de l'île après avoir obligé le tiers de la population de Chypre à se réfugier dans la partie libre. Face aux sanctions répétées imposées à la Russie pour sa politique en Ukraine, ce sentiment d'amertume s'est d'ailleurs encore renforcé.

\section{RÉSUMÉS}

Dans l'article qui suit, nous allons tenter d'approcher un phénomène très récent qui s'attache aux réalités culinaires de l'île de Chypre et qui n'a pas encore été étudié. Il s'agit de la présence à Chypre d'un nombre croissant de restaurants et de bistrots grecs, notamment depuis une quinzaine d'années, phénomène lié en partie à la prospérité et au niveau de vie plus élevés à Chypre qu'en Grèce, du moins jusqu'en 2013, ainsi qu'à la crise durable de l'économie grecque depuis la fin de la dernière décennie. Les conclusions de cette approche pourraient être utiles pour une analyse plus approfondie de la question. 